\title{
Apsidal motion and absolute parameters for five LMC eccentric eclipsing binaries ${ }^{\star} \star \star \star$
}

\author{
P. Zasche and M. Wolf
}

\author{
Astronomical Institute, Charles University in Prague, Faculty of Mathematics and Physics, 18000 Praha 8, V Holešovičkách 2, \\ Czech Republic \\ e-mail: zasche@sirrah.troja.mff.cuni.cz
}

Received 11 June 2013 / Accepted 15 July 2013

\begin{abstract}
Aims. As part of our observational projects at the La Silla Danish 1.54-meter telescope, we aim to measure the precise times of minimum light for eccentric eclipsing binaries in the Large Magellanic Cloud, needed for accurate determination of apsidal motion. Many new times of minima were derived from the photometric databases OGLE and MACHO. Several new minima were also observed. Five early-type and eccentric-orbit eclipsing binaries: HV $982(P=5.34, e=0.15)$, HV $2274(5.73,0.17)$, MACHO 78.6097.13 (3.11,0.05), MACHO 81.8881.47 (3.88, 0.22), and MACHO 79.5377.76 (2. $64,0.06)$ were studied.

Methods. The $\mathrm{O}-\mathrm{C}$ diagrams of the systems were analysed using all reliable timings found in the literature, and new or improved elements of apsidal motion were obtained. Light and radial velocity curves of MACHO 81.8881.47 and MACHO 79.5377.76 were analysed using the program PHOEBE.

Results. We derived for the first time or significantly improved the relatively short periods of apsidal motion of 211 (12), 127 (8), 48 (13), 103 (20), and 42 (19) years, respectively. The internal structure constants, $\log k_{2}$, were found to be $-2.37,-2.47,-2.17,-2.02$, and -1.86 respectively, under the assumption that the component stars rotate pseudosynchronously. The relativistic effects are weak, up to $6 \%$ of the total apsidal motion rate. The masses for MACHO 81.8881.47 resulted in $5.51(0.21)$ and $5.40(0.19) M_{\odot}$, while for MACHO 79.5377 .76 the masses are $11.26(0.35)$ and $11.27(0.35) M_{\odot}$, respectively.
\end{abstract}

Key words. binaries: eclipsing - stars: early-type - stars: general - stars: fundamental parameters - Magellanic Clouds

\section{Introduction}

Eccentric eclipsing binaries (EEB) with an apsidal motion can provide us with an important observational test of theoretical models of stellar structure and evolution. A long-term collecting the times of minima of EEBs observed throughout the apsidal motion cycle and consecutive detailed analysis of the period variations of EEB can be performed, yielding both the orbital eccentricity and the period of rotation of the apsidal line with high accuracy (Giménez 1994). Many different sets of stellar evolution models have been published in recent years, e.g. Maeder (1999), Claret (2004), or Claret (2006); however, to distinguish between them and to test which one is more suitable is still rather difficult. The internal structure constants as derived from the apsidal motion analysis could serve as one independent criterion. On the other hand, to discriminate between the models only stellar parameters for EEBs with an accuracy of $1 \%$ can be used.

The Magellanic Clouds are of prime importance in the context of stellar evolution theory. However, the chemical composition of the Magellanic Clouds differs from that of the solar neighborhood (e.g. Ribas 2004) and the study of these massive and metal-deficient stars in the LMC checks our evolutionary models for these abundances. All eclipsing binaries analysed here

* Based on observations made with ESO Telescopes at the La Silla Paranal Observatory under programme ID 68.A-0223(A), and on data collected with the Danish $1.54 \mathrm{~m}$ telescope at the ESO La Silla Observatory.

$\star \star$ Appendices are available in electronic form at http://www . aanda.org have properties that make them important astrophysical laboratories for studying the structure and evolution of massive stars (Ribas 2004).

Here we analyse the observational data and rates of apsidal motion for five LMC detached eclipsing systems. All these systems are early-type objects known to have eccentric orbits and to exhibit apsidal motion. Similar studies of LMC EEBs have been presented by Michalska \& Pigulski (2005, hereafter MP05) and Michalska (2007).

\section{Observations of minimum light}

The monitoring of faint EEBs in external galaxies requires only moderate telescopes in the 1-2 m class range equipped with a modern CCD camera. However, a large amount of observing time is needed, which is unavailable at larger telescopes. During past observational seasons, we accumulated over 1600 photometric observations at selected phases during primary and secondary eclipses and derived 16 precise times of minimum light for selected eccentric systems. New CCD photometry was obtained at the La Silla Observatory in Chile, where the $1.54 \mathrm{~m}$ Danish telescope (hereafter DK154) with the CCD camera and $R I$ filters was used.

All CCD measurements were dark-subtracted and then flatfielded using sky exposures taken at either dusk or dawn. Several comparison stars were chosen in the same frame as the variables. A synthetic aperture photometry and astrometry software developed by Velen and Pravec called APHOT, was routinely used for data obtained. No correction for differential extinction was 
Table 1. Apsidal motion elements for HV 982, HV 2274, MACHO 78.6097.13, MACHO 81.8881.47, and MACHO 79.5377.76.

\begin{tabular}{lccccc}
\hline \hline Element [Unit] & HV 982 & HV 2274 & MACHO 78.6097.13 & MACHO 81.8881.47 & MACHO 79.5377.76 \\
\hline$T_{0}$ [HJD] & $2449340.5146(195)$ & $2448099.8540(9)$ & $2452424.6888(78)$ & $2452282.2431(178)$ & $2452262.6776(67)$ \\
$P_{\mathrm{s}}$ [days] & $5.3352210(97)$ & $5.7259971(8)$ & $3.1070278(104)$ & $3.8818717(32)$ & $2.6365767(79)$ \\
$P_{\mathrm{a}}$ [days] & $5.3355902(97)$ & $5.7267045(8)$ & $3.1075678(104)$ & $3.8822732(33)$ & $2.6370342(79)$ \\
$e$ & $0.149(0.033)$ & $0.1252(39)$ & $0.0459(139)$ & $0.217(23)$ & $0.0574(160)$ \\
$\dot{\omega}\left[\mathrm{deg}\right.$ cycle $\left.^{-1}\right]$ & $0.0249(0.0014)$ & $0.0445(29)$ & $0.0633(264)$ & $0.03723(606)$ & $0.0625(194)$ \\
$\dot{\omega}\left[\mathrm{deg} \mathrm{yr}^{-1}\right]$ & $1.705(0.095)$ & $2.84(0.20)$ & $7.44(3.1)$ & $3.50(0.57)$ & $8.65(2.68)$ \\
$\omega_{0}[\mathrm{deg}]$ & $221.2(0.7)$ & $71.9(2.9)$ & $27.9(4.0)$ & $95.9(8.6)$ & $31.57(4.20)$ \\
$U$ [yr] & $211(12)$ & $126.9(7.9)$ & $48.4(12.5)$ & $102.8(20.0)$ & $41.6(18.8)$ \\
\hline
\end{tabular}

applied because of the proximity of the comparison stars to the variable and the resulting negligible differences in air mass and their similar spectral types.

The new times of primary and secondary minima and their errors were determined by the classical Kwee-van Woerden (1956) algorithm. All new times of minima are given in Table A.1, where epochs are calculated from the ephemeris given in Table 1; the other columns are self-evident.

\section{Photometry}

For all of the systems we harvested the MACHO (Faccioli et al. 2007) and OGLE (Graczyk et al. 2011) photometry available online. These photometric data were used both for minima times analysis as well as for light curve analysis.

The analysis of the light curves for two of the systems was carried out using the program PHOEBE, ver. 0.31a (Prša \& Zwitter 2005), which is based on the Wilson-Devinney algorithm (Wilson \& Devinney 1971) and its later modifications, but some of the parameters had to be fixed during the fitting process. The albedo coefficient remained fixed at value 1.0 and the gravity darkening coefficients $g=1.0$. The limb darkening coefficients were interpolated from the van Hamme tables (van Hamme 1993). A problem emerged with the synchronicity parameters $\left(F_{i}\right)$ due to poor coverage of the RV data near the eclipses and low quality of the light curves used for the LC analysis, hence we fixed these values at $F_{i}=0$. The temperature of the primary component was derived from the $V-I$ photometric index, from MP05 and from the resulting masses derived from the combined $\mathrm{LC}+\mathrm{RV}$ analysis.

\section{Spectroscopy}

The spectroscopic data for two of the systems (MACHO 81.8881.47, MACHO 79.5377.76) were found in the ESO Archive of the UV-Visual Echelle Spectrograph (UVES) at the Very Large Telescope (VLT). The spectra were obtained during the ESO Period 68 program "Precise distances to the LMC and SMC from double-lined eclipsing binaries"; the PI of the project was A. Clausen. Typical exposure times were about 1500 seconds, while the spectra typically have a signal-to-noise ratio $(\mathrm{S} / \mathrm{N})$ of about 50 . The original data were reduced using the standard ESO routines. The final radial velocities (hereafter RV) used for the analysis were derived via a manual cross-correlation technique (i.e. the direct and flipped profile of spectral lines manually shifted on the computer screen to achieve the best match) using program SPEFO (Horn et al. 1996; Škoda 1996) on several absorbtion lines in the measured spectral region (usually $\mathrm{H}_{\beta}$ to $\mathrm{H}_{\vartheta}$ ). The derived radial velocities are given in Table B.1. We estimate the error of individual data points to be about $5 \mathrm{~km} \mathrm{~s}^{-1}$.

\section{An approach for the analysis}

For the analysis we used the approach presented below. For the systems where the inclination of the orbit is known, the first two steps can be skipped.

- First, all of the available photometric data were analysed, resulting in a set of minima times. Preliminary apsidal motion parameters were derived (with the assumption $i=90^{\circ}$ ).

- Second, the eccentricity $(e)$, argument of periastron $(\omega)$, and apsidal motion rate $(\dot{\omega})$ derived from the apsidal motion analysis were used for the preliminary light curve (hereafter LC) analysis.

- Third, the inclination ( $i$ ) from the LC analysis was used for the final apsidal motion analysis.

- And finally, the resulting $e, \omega$, and $\dot{\omega}$ values from the apsidal motion analysis were used for the final LC + RV analysis.

\section{Apsidal motion analysis}

The apsidal motion in all eccentric systems was studied by means of an $\mathrm{O}-\mathrm{C}$ diagram analysis. All available times of minima, both published in the literature and newly measured, were analysed using the method presented by Giménez \& García-Pelayo (1983). This is a weighted least-squares iterative procedure, including terms in the eccentricity up to the fifth order. There are five independent variables $\left(T_{0}, P_{\mathrm{s}}, e, \dot{\omega}, \omega_{0}\right)$ determined in this procedure. The periastron position $\omega$ is given by the linear equation

$\omega=\omega_{0}+\dot{\omega} E$

where $\dot{\omega}$ is the rate of periastron advance, and the position of periastron for the zero epoch $T_{0}$ is denoted as $\omega_{0}$. The relation between the sidereal and the anomalistic period, $P_{\mathrm{s}}$ and $P_{\mathrm{a}}$, is given by

$P_{\mathrm{s}}=P_{\mathrm{a}}\left(1-\dot{\omega} / 360^{\circ}\right)$,

and the period of apsidal motion by

$U=360^{\circ} P_{\mathrm{a}} / \dot{\omega}$.

All new precise CCD times of minima were used with a weight of 10 or 20 in our computation. Some of our less precise measurements were weighted by a factor of 5 , while the earlier visual and photographic times (esp. the times of the mid-exposure of a photographic plate) were given a weight of one or nought because of the large scatter in these data. 


\section{Analysis of the systems}

\subsection{HV 982}

The detached eclipsing binary HV 982 (also known as MACHO 82.8043.26, LMV110; $V_{\max }=14^{\mathrm{m}} .65 ; \mathrm{Sp}$. B5V) is a relatively well-known LMC binary with an eccentric orbit $(e=0.15)$ and a moderate orbital period of 5.3 days. It was discovered to be a variable star by Gaposhkin (1970), who published the first photographic light curve.

Pritchard et al. $(1994,1998)$ in their photometric study derived high surface temperatures $(\sim 28000 \mathrm{~K})$ and masses $\left(\sim 8 M_{\odot}\right)$ of components. They also derived the apsidal motion period $U=205 \pm 7$ years. The precise stellar parameters of components of HV 982 were derived spectroscopically by Fitzpatrick et al. (2002), who found components with similar mass and size,

$M_{1}=11.28 \pm 0.46 M_{\odot}, M_{2}=11.61 \pm 0.47 M_{\odot}$,

$R_{1}=7.15 \pm 0.12 R_{\odot}, R_{2}=7.92 \pm 0.13 R_{\odot}$.

The following linear light elements were given in that paper:

Pri. Min. = HJD $2449340 \mathrm{~d} 7172+5.33522 \times \mathrm{E}$.

Using the complete analyses of HV 982 they also found the distance to the center of the $\mathrm{LMC} d_{\mathrm{LMC}}=50.7 \pm 1.2 \mathrm{kpc}$. Later, Clausen et al. (2003) presented a new accurate CCD uvby light curve obtained at the Danish $1.54 \mathrm{~m}$ telescope at La Silla and derived the precise photometric elements with apsidal motion period $U=208 \pm 15$ years.

All CCD times of minimum light given in Pritchard et al. (1998) and Clausen et al. (2003) were incorporated into our analysis. Using MACHO (Faccioli et al. 2007) and OGLE (Graczyk et al. 2011) photometry, we were able to derive additional times of minimum light. A total of 68 times of minimum light were used in our analysis (see Table A.1). The orbital inclination adopted was $i=89^{\circ} .3$, based on the analysis of Fitzpatrick et al. (2002). The computed apsidal motion parameters and their internal errors of the least-squares fit are given in Table 1 . In this table, $P_{\mathrm{s}}$ denotes the sidereal period, $P_{\mathrm{a}}$ the anomalistic period, $e$ represents the eccentricity, and $\dot{\omega}$ is the rate of periastron advance (in degrees per cycle and in degrees per year). The zero epoch is given by $T_{0}$, and the corresponding position of the periastron is represented by $\omega_{0}$. The $\mathrm{O}-\mathrm{C}$ residuals for all times of minimum with respect to the linear part of the apsidal motion equation (Min $\left.=T_{0}+P \times E\right)$ are shown in Fig. 1 . The non-linear predictions, corresponding to the fitted parameters, are plotted for primary and secondary eclipses.

\subsection{HV 2274}

The detached and double-lined eclipsing binary HV 2274 (also known as MACHO 19.3577.7, 2MASS J05024076-6824212, LMV 182, FL 3556; $V_{\max }=14^{\mathrm{m}} .13$; Sp. B1-2 IV-III) is a relatively bright and well-studied LMC eclipsing binary with an eccentric orbit ( $e=0.17$ ) and a moderate orbital period of 5.7 days. It was discovered to be a variable star by Leavitt (1908). Later, Shapley \& Nail (1953) recognized its eclipsing nature and classified it as a $\beta$ Lyrae type. The eccentric orbit and apsidal motion of HV 2274 was first announced by Watson et al. (1992), who obtained the $B V I_{\mathrm{c}} \mathrm{CCD}$ photometry at the Mount John University Observatory. See also the history of HV 2274 in the last paper mentioned. Later Claret (1996) found the masses and evolutionary status of this system:

Pri. Min. = HJD 2448 099. $818+$ 5.726006 $\times$ E.

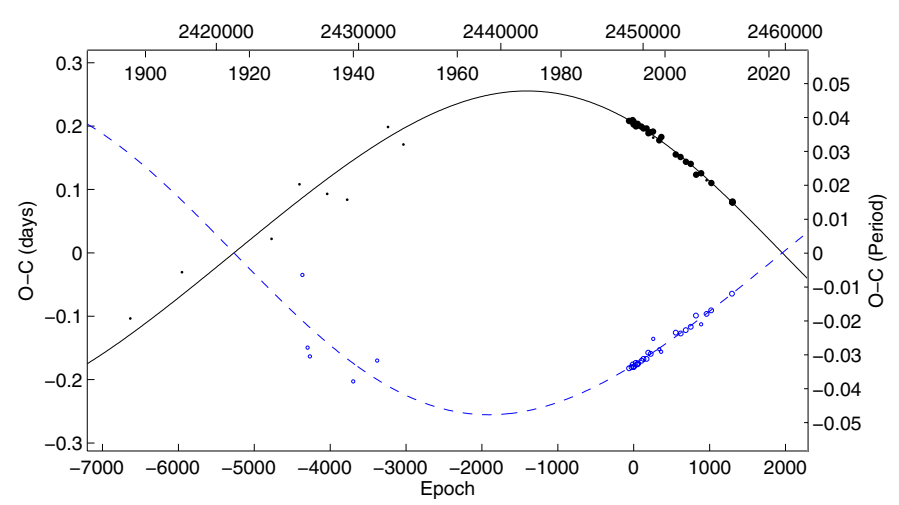

Fig. 1. O-C diagram for the times of minima of HV 982. The continuous and dashed curves represent predictions for the primary and secondary eclipses, respectively. The individual primary and secondary minima are denoted by dots and open circles, respectively. Larger symbols correspond to the photoelectric or CCD measurements that were given higher weights in the calculations.

The fundamental properties of HV 2274 were most recently given in Ribas et al. (2000) who determined the precise absolute parameters of both eclipsing components to be

$M_{1}=12.2 \pm 0.7 M_{\odot}, M_{2}=11.4 \pm 0.7 M_{\odot}$,

$R_{1}=9.86 \pm 0.24 R_{\odot}, R_{2}=9.03 \pm 0.24 R_{\odot}$.

They also derived the improved apsidal motion period $U=$ $123 \pm 3$ years and the value of internal structure constant log $k_{2, \text { obs }}=-2.56$. Since the above-mentioned papers were published, new times of minima have been obtained, which allowed us to reduce the uncertainties in the derived parameters. We collected all times of minimum light given in the literature together with new ones derived from MACHO, OGLE, and our new photometry obtained in Chile. All of these values are listed in Table A.1. In total, 58 precise times of minimum light were used in our analysis, including 29 secondary eclipses. The orbital inclination adopted was $i=89^{\circ} .6$, based on the analysis of Ribas et al. (2000).

Analysing the available data using the apsidal motion hypothesis, we found an additional variation superimposed on the apsidal motion. Hence, we used a different code computing the apsidal motion parameters together with the third-body orbit (a so-called light travel time effect), see e.g. Irwin (1959). Altogether, ten parameters were fitted (five from apsidal motion, five from the third body hypothesis), thus this approach led to an acceptable solution. The resulting parameters of the fit are given in Tables 1 and 2, the complete $\mathrm{O}-\mathrm{C}$ diagrams are shown in Figs. 2 and 3. From the third-body parameters we were also able to compute the mass function of the distant component, which resulted in $f\left(m_{3}\right)=0.053 \pm 0.008 M_{\odot}$. From this value, we calculated the predicted minimal mass of the third body (i.e. assuming coplanar orbits $i_{3}=90^{\circ}$ ), which resulted in $m_{3, \min }=3.4 \mathrm{M}_{\odot}$. If we propose this body in the system, one can ask whether it is detectable somehow in the already-obtained data. The period is rather long for continuous monitoring of the radial velocity changes, but detecting the third light in the light curve solution is difficult. Assuming a normal main sequence star, its luminosity is about only $1 \%$ of the total system luminosity. Such a weak third light could be detectable only in extremely precise photometric data for the light curve solution. 
Table 2. Third-body orbit parameters for HV 2274.

\begin{tabular}{lc}
\hline \hline Element [Unit] & Value \\
\hline$p_{3}$ [yr] & $98.2 \pm 14.3$ \\
$A_{3}$ [day] & $0.045 \pm 0.009$ \\
$T_{3}$ [HJD] & $2456205 \pm 5033$ \\
$e_{3}$ & $0.654 \pm 0.047$ \\
$\omega_{3}$ [deg] & $251.1 \pm 7.1$ \\
\hline
\end{tabular}

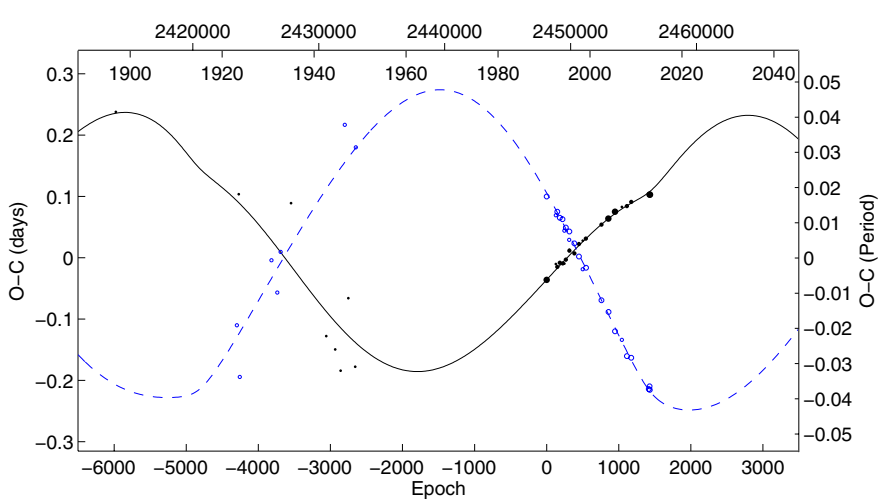

Fig. 2. O-C diagram for the times of minima of HV 2274. See legend to Fig. 1. The final fit is composed from two effects: the apsidal motion together with the third-body hypothesis.

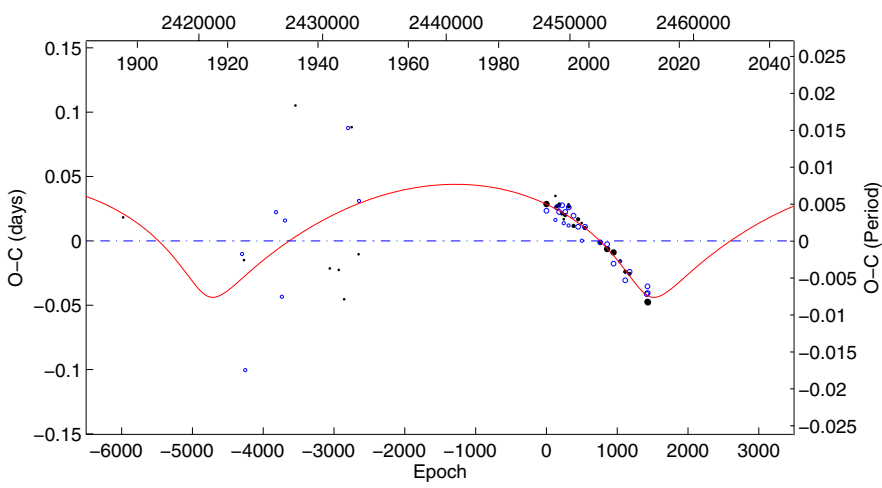

Fig. 3. O-C residuals for HV 2274 after subtraction of the apsidal motion term. Only the third body effect is plotted.

\section{3. $\mathrm{MACHO} 78.6097 .13$}

The detached eclipsing binary MACHO 78.6097.13 (also known as OGLE J051804.81-694818.9, LMC MP\#5; $V_{\max }=14$ m.37, sp $09 \mathrm{~V}+\mathrm{O} 9 \mathrm{~V})$ is an eccentric binary system $(e=0.046)$ with a short orbital period $(P=3.1 \mathrm{~d})$ discovered by MP05 in the OGLE field LMC-SC7.

Our analysis gives the following ephemeris:

Pri. Min. $=$ HJD $2452424^{\mathrm{d}} .6888+3$ d. $1070278 \times$ E.

The most detailed analysis of the system was published by González et al. (2005), which was based on the ESO spectral observations together with the MACHO data. They also derived the masses and radii of both components that were used in the present analysis. Both components are of O9 spectral types, which makes this system the earliest in our sample.

Our new times of minimum light, as well as the timings derived from MACHO and OGLE photometry are given in Table A.1. All of these data (31 minima times) were used in our calculations. Using the parameters presented by González et al. (2005),

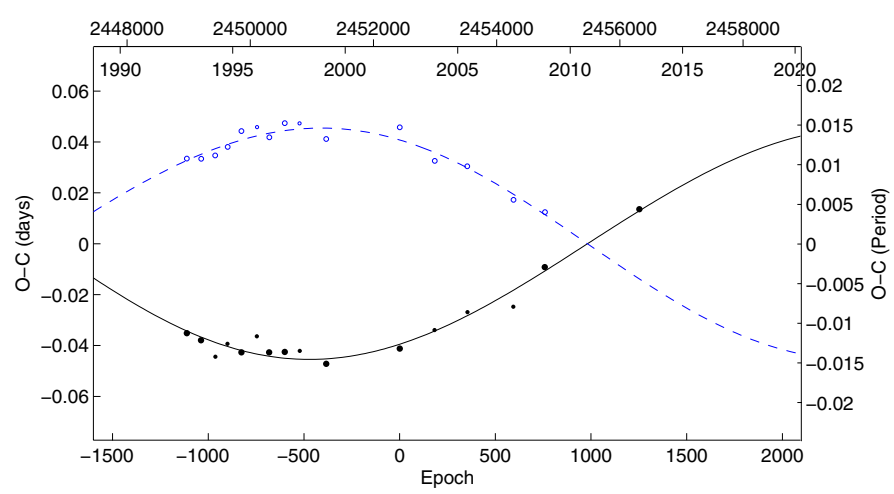

Fig. 4. O-C graph for the times of minimum of MACHO 78.6097.13. See legend to Fig. 1.

we analysed the system on apsidal motion, deriving the parameters given in Table 1. As one can see, this system has the lowest eccentricity in our sample and the apsidal advance is rather fast: almost one half of the period has been covered with observations so far.

\subsection{MACHO 81.8881.47}

The detached eclipsing binary MACHO 81.8881.47 (also known as OGLE J053517.75-694318.7, LMC MP\#7; $V_{\max }=14$ m9; $\mathrm{Sp} . \mathrm{B}$ ) is a relatively bright binary system with an eccentric orbit $(e=0.2)$ and a moderate orbital period $P \simeq 3.9$ days. Its variability was discovered by MP05 in the OGLE field LMC-SC16. Most recently, Graczyk et al. (2011) included this star in their catalogue of eclipsing binaries in the LMC. They also gave the preliminary ephemeris:

Pri. Min. = HJD $2453571.1063+3.881980 \times$ E.

Using OGLE and MACHO photometry, we were also able to derive additional times of minimum light; two more minima were derived from our observations. Only 30 times of minimum light were used in our analysis (see Table A.1). As one can clearly see from the shape of the light curve and the different duration of both the primary and secondary eclipse, this is the system with the highest value of eccentricity in our sample.

We used the ESO data for deriving the radial velocities of the components. Ten UVES spectra were found, of which nine were usable for the analysis. The derived RVs of both components are given in Table B.1. Together with these radial velocities, the MACHO light curve was used for the subsequent combined LC+RV analysis. The final parameters of the solution are given in Table 3, while the fits are plotted in Figs. 5 and 6. Our results show the rather noticeable property that the more massive component (the primary) is smaller and less luminous (i.e. has a lower temperature). However, this is still a preliminary result based on only nine RVs and a poor fit. Although the scenario is possible, the more probable explanation is that the mass ratio is inverse, and the primary and secondary components are interchanged. This can be allowed for within the uncertainties of the mass ratio. A more detailed analysis is needed, based on more precise spectral observations. The orbital inclination was about $i=84^{\circ}$.2, which was later used for the apsidal motion analysis. The resulting parameters of apsidal motion are found in Table 1, and the current $\mathrm{O}-\mathrm{C}$ diagram is shown in Fig. 7. As one can see, the period is rather long and only about $1 / 5$ has been covered so far. 
P. Zasche and M. Wolf: Apsidal motion in five LMC eclipsing binaries

Table 3. Light and radial velocity curve fit parameters for MACHO 81.8881.47 and MACHO 79.5377.76.

\begin{tabular}{lcc}
\hline \hline Parameter & MACHO 81.8881.47 & MACHO 79.5377.76 \\
\hline$T_{1}[\mathrm{~K}]$ & 17200 (fixed) & $27500($ fixed) \\
$T_{2}[\mathrm{~K}]$ & $18420(360)$ & $26710(470)$ \\
$i[\mathrm{deg}]$ & $84.20(0.27)$ & $87.22(0.44)$ \\
$\Omega_{1}$ & $7.067(0.115)$ & $5.774(0.085)$ \\
$\Omega_{2}$ & $6.365(0.102)$ & $6.264(0.098)$ \\
$q=M_{2} / M_{1}$ & $0.981(0.030)$ & $1.00(0.02)$ \\
$a\left[R_{\odot}\right]$ & $23.04(0.12)$ & $22.65(0.07)$ \\
$v_{\gamma}\left[\mathrm{km} \mathrm{s}^{-1}\right]$ & $272.6(1.6)$ & $250.0(1.0)$ \\
$L_{1}[\%]$ & $41.4(1.2)$ & $57.2(1.5)$ \\
$L_{2}[\%]$ & $58.6(1.4)$ & $42.8(1.1)$ \\
\hline
\end{tabular}

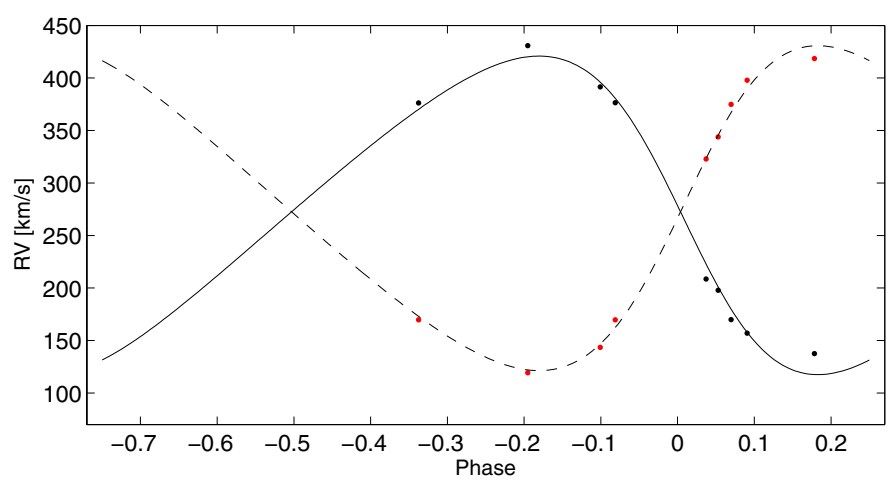

Fig. 5. Radial velocity curve for MACHO 81.8881.47.

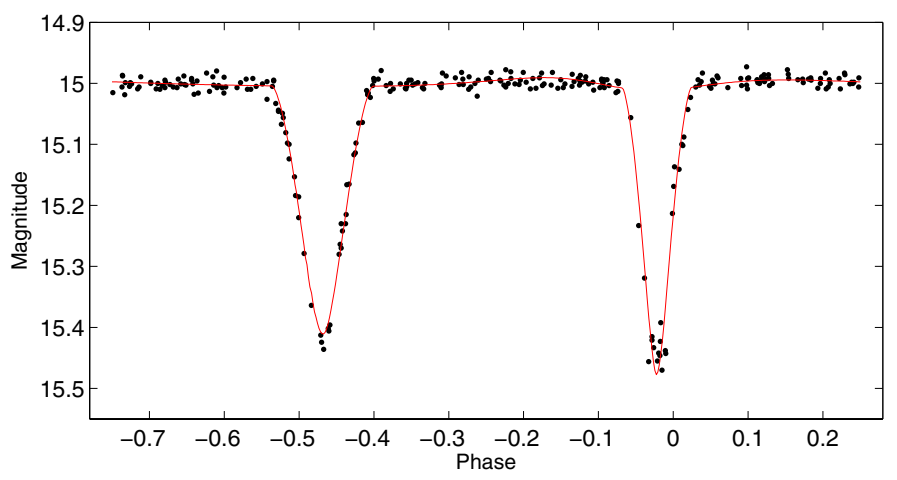

Fig. 6. Light curve for MACHO 81.8881.47.

\subsection{MACHO 79.5377.76}

The detached eclipsing binary MACHO 79.5377.76 (also known as OGLE J051323.98-692249.2, $V_{\max }=15^{\mathrm{m}} .8$; Sp. B) is a fairly neglected binary system with a moderate eccentric orbit $(e=$ $0.06)$ and a short orbital period $(P \simeq 2.64$ day):

Pri. Min. = HJD $2452262.6776+2$ d. $6365767 \times$ E.

We used the MACHO and OGLE photometry together with our new observations from Chile to derive the times of minima, the ESO spectral observations to derive the radial velocities, and the MACHO photometry to model the light curve of the system. Our results are plotted in Figs. 8 and 9. The resulting parameters are given in Table 3 . As one can see, both components are of equal mass and their spectral type was estimated to be about B0-B1. Radial velocities used for our analysis are also given in Table B.1. The coverage of the radial velocity curve is better than for MACHO 81.8881.47; however, it was rather difficult to

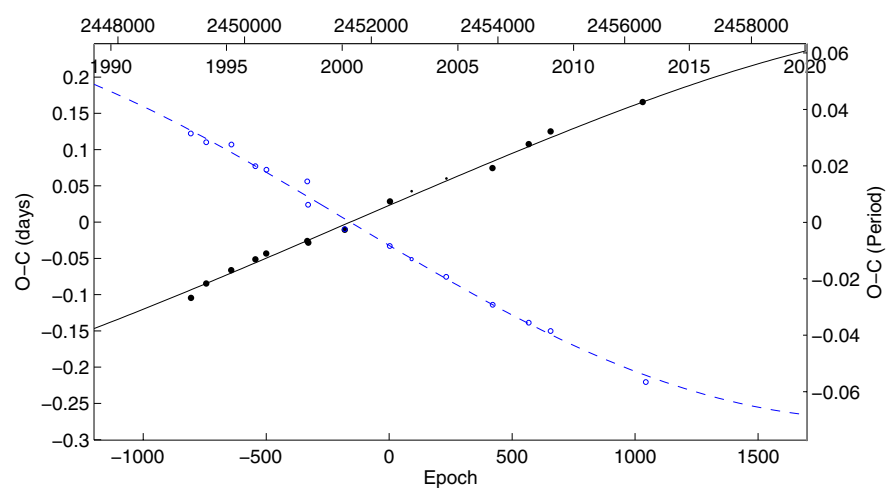

Fig. 7. O-C graph of MACHO 81.8881.47. See legend for Fig. 1.

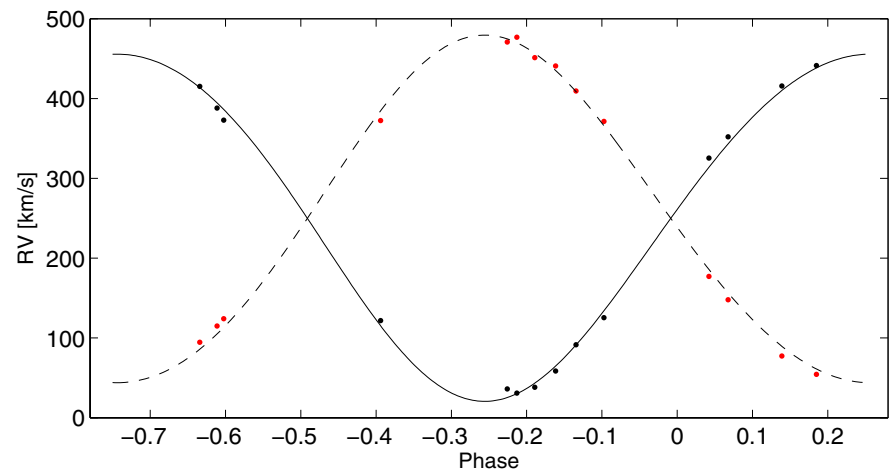

Fig. 8. Radial velocity curve for MACHO 79.5377.76.

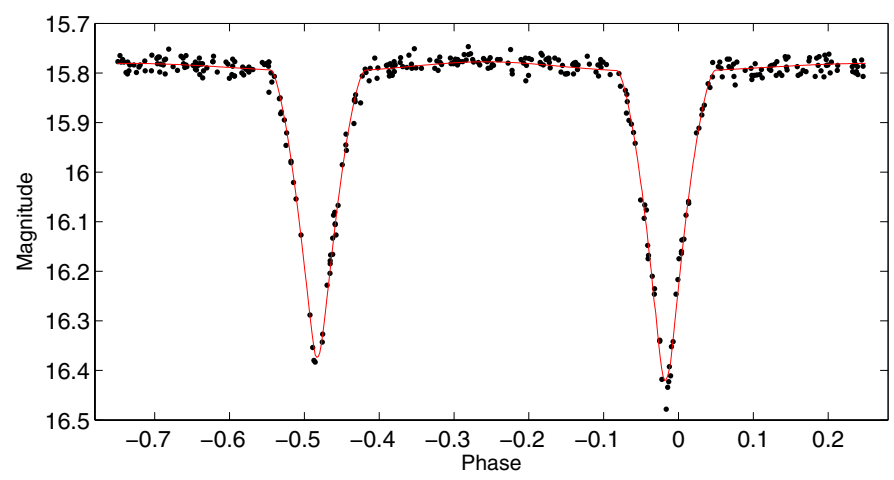

Fig. 9. Light curve for MACHO 79.5377.76.

derive the synchronicity parameters $F_{i}$, and so we fixed them at $F_{i}=0$. A total of 34 reliable times of minimum light were used in our analysis including 16 secondary eclipses (see tables in the Appendices). The final apsidal motion elements are given in Table 1, and the $\mathrm{O}-\mathrm{C}$ graph is shown in Fig. 10. The apsidal period is the shortest among systems studied here, only 42 years.

\section{Discussion}

The detection of apsidal motion in EEB provides the opportunity to test models of stellar internal structure. The internal structure constant (ISC) $k_{2, \text { obs }}$ is related to the variation in the density inside the star and can be derived using the expression

$k_{2, \mathrm{obs}}=\frac{1}{c_{21}+c_{22}} \frac{P_{\mathrm{a}}}{U}=\frac{1}{c_{21}+c_{22}} \frac{\dot{\omega}}{360}$, 
Table 4. Basic physical properties of HV 982, HV 2284, MACHO 78.6097.13, MACHO 81.8881.47, and MACHO 79.5377.76 and their internal structure constants.

\begin{tabular}{lcccccc}
\hline \hline Parameter & Unit & HV 982 & HV 2274 & MACHO 78.6097.13 & MACHO 81.8881.47 & MACHO 79.5377.76 \\
\hline$M_{1}$ & $M_{\odot}$ & $11.28(0.46)$ & $12.2(0.7)$ & $11.76(0.48)$ & $5.51(0.21)$ & $11.26(0.35)$ \\
$M_{2}$ & $M_{\odot}$ & $11.61(0.47)$ & $11.4(0.7)$ & $10.51(0.40)$ & $5.40(0.19)$ & $11.27(0.35)$ \\
$r_{1}$ & & $0.194(0.003)$ & $0.255(0.013)$ & $0.258(0.021)$ & $0.169(0.008)$ & $0.212(0.004)$ \\
$r_{2}$ & & $0.214(0.003)$ & $0.234(0.012)$ & $0.206(0.020)$ & $0.192(0.010)$ & $0.190(0.004)$ \\
\hline Source & & Clausen et al. & Ribas et al. & González et al. & This & This \\
& & $(2003)$ & $(2000)$ & $(2005)$ & paper & paper \\
\hline$\dot{\omega}_{\text {rel }}$ & deg cycle & 0.0015 & 0.0014 & 0.0020 & 0.0011 & 0.0023 \\
$\dot{\omega}_{\text {rel }} / \dot{\omega}$ & $\%$ & 5.9 & 3.2 & 3.24 & 3.06 & $-2.017(0.18)$ \\
$\log k_{2, \text { obs }}$ & & $-2.371(0.10)$ & $-2.470(0.15)$ & $-2.174(0.35)$ & -2.01 & $-1.859(0.17)$ \\
$\log k_{2, \text { theo }}$ & & -2.32 & -2.45 & -2.19 & -1.90 & \\
\hline
\end{tabular}

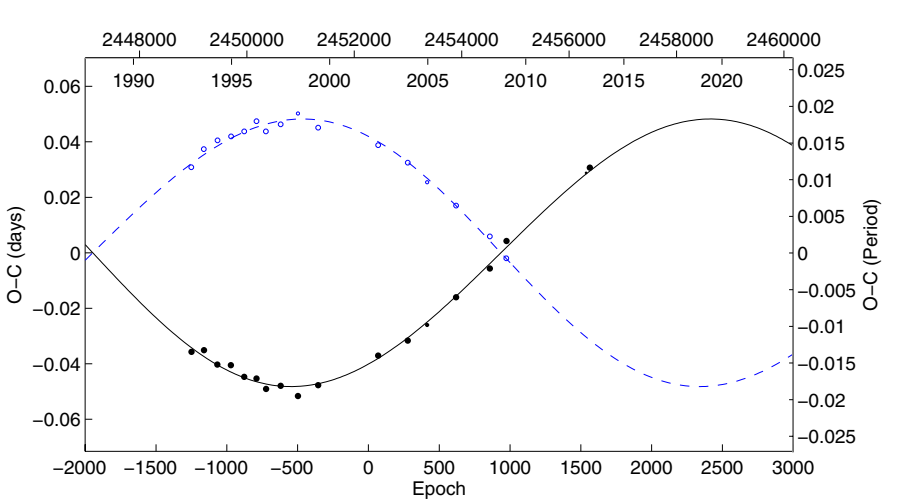

Fig. 10. O-C diagram for MACHO 79.5377.76. See legend to Fig. 1.

where $c_{21}$ and $c_{22}$ are functions of the orbital eccentricity, fractional radii, the masses of the components, and the ratio between rotational velocity of the stars and Keplerian velocity (Kopal 1978). We also assume that the component stars rotate pseudosynchronously with the same angular velocity as the maximum orbital value at periastron (see e.g. Kopal 1978). Another possible approach is to use the value of $v \sin i$ as derived from the combined LC+RV analysis published earlier. However, there could be a problem with the inclination of the rotation axis (as in the case of DI Her) and, moreover, the error of the internal structure constants is by far dominated by the term $r_{i}^{5}$ in the equations. In addition to the classical Newtonian contribution, the observed rate of rotation of the apses includes the contribution from General Relativity (Giménez 1985),

$\dot{\omega}_{\text {rel }}=5.45 \times 10^{-4} \frac{1}{1-e^{2}}\left(\frac{M_{1}+M_{2}}{P}\right)^{2 / 3}$,

where $M_{i}$ denotes the individual masses of the components in solar units and $P$ is the orbital period in days.

The values of $\dot{\omega}_{\text {rel }}$ and the resulting mean internal structure constants $k_{2, \text { obs }}$ for the systems studied are given in Table 4. Theoretical values $k_{2 \text {,theo }}$ according to available theoretical models for the internal stellar structure computed by Claret (2006) for given masses of components are presented in Table 4.

We tried to compare our resulting systemic velocities with other published values of eclipsing binaries in the LMC, see Table 5 and Fig. 11. There are still only a few such systems studied in detail (i.e. LC+RV analysis). Therefore, reliable analysis of different velocities within the LMC is still very difficult. We can only compare our Fig. 11 with other kinematic studies of the LMC published earlier, e.g. that by Reid \& Parker (2006)

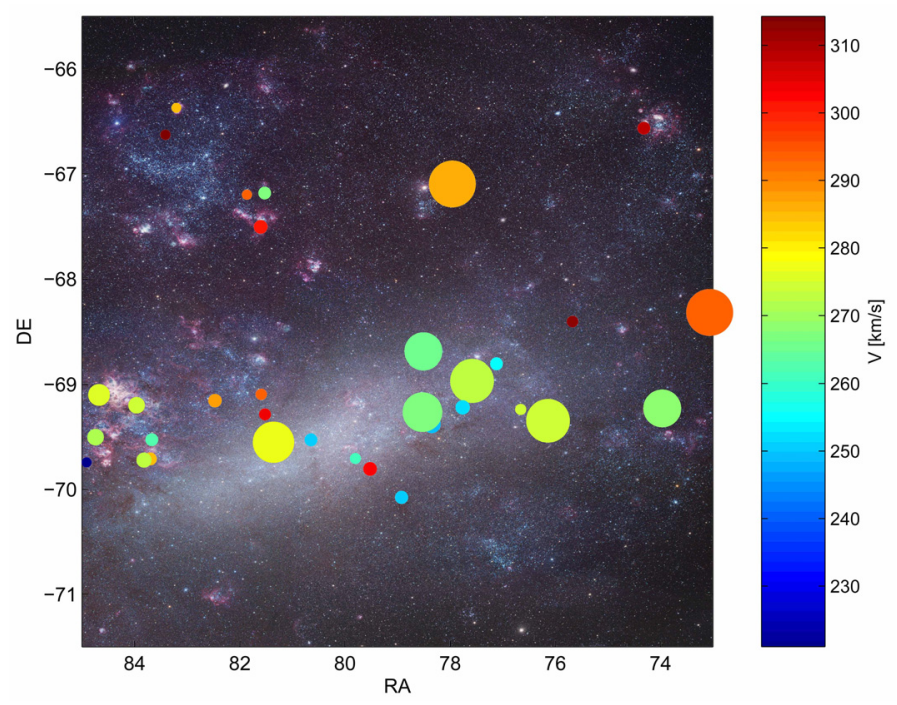

Fig. 11. Position and systemic velocities (see the colour scale on the right) of eclipsing binaries located in the LMC and its vicinity, see Table 5. The larger the symbol, the higher the precision. The background image of the LMC is used with the permission of the author Robert Gendler (http://www.robgendlerastropics.com).

or Rohlfs et al. (1984), that were based on much larger radialvelocity data sets. Nevertheless, we can conclude that our two new systemic velocities roughly fit the overall picture and the total velocity dispersion within the LMC is more than $30 \mathrm{~km} \mathrm{~s}^{-1}$.

\section{Conclusions}

The apsidal motion in EEB has been used for decades to test evolutionary stellar models. This study provides accurate information on the apsidal motion rates of five mainsequence early-type binary systems in the LMC: HV 982, HV 2274, MACHO 78.6097.13, MACHO 81.8881.47, and MACHO 79.5377.76. In our Galaxy there are known a few hundreds of apsidal motion EEBs, however in the LMC there are still only a few dozen of these systems (Michalska \& Pigulski 2005). Hence this study still presents an important contribution to the topic. The relativistic effects are weak, being up to $6 \%$ of the total apsidal motion rate. For the systems MACHO 79.5377.76 and MACHO 81.8881.47, their light and radial velocity curves were analysed for the first time yielding the stellar parameters of both components given in Table 4. Moreover, when the MACHO 79.5377.76 internal structure constant and the 
Table 5. Radial velocities of eclipsing binaries in the LMC derived from various analyses.

\begin{tabular}{|c|c|c|c|c|c|}
\hline System & $\mathrm{RA}[\mathrm{h} \mathrm{m} \mathrm{s}]$ & $\mathrm{DE}\left[{ }^{0}, \prime \prime \prime\right]$ & $P[\mathrm{~d}]$ & $\mathrm{RV}\left[\mathrm{km} \mathrm{s}^{-1}\right]$ & Reference \\
\hline MACHO 47.1884.17 & 045215.29 & -681910.55 & 251.0068 & $293.44 \pm 0.04$ & Pietrzyński et al. (2013) \\
\hline MACHO 18.2475 .67 & 045551.48 & -691347.99 & 150.0198 & $267.68 \pm 0.08$ & Pietrzyński et al. (2013) \\
\hline HV 2241 & 045715.74 & -663354.20 & 4.342635 & $307.9 \pm 3$ & Ostrov et al. (2001) \\
\hline HV 2274 & 050240.80 & -682421.02 & 5.726006 & $312 \pm 4$ & Ribas et al. (2000) \\
\hline MACHO 1.3926 .29 & 050432.88 & -692050.99 & 189.8215 & $274.32 \pm 0.05$ & Pietrzyński et al. (2013) \\
\hline MACHO 1.4290 .113 & 050637.80 & -691422.98 & 2.273210 & $274.2 \pm 4.5$ & González et al. (2005) \\
\hline MACHO 1.4539 .37 & 050828.10 & -684826.02 & 2.995450 & $255.6 \pm 3.1$ & González et al. (2005) \\
\hline OGLE LMC-ECL-9114 & 051019.65 & -685812.0 & 214.1707 & $272.04 \pm 0.05$ & Pietrzyński et al. (2013) \\
\hline МАCHO 79.5017.83 & 051102.80 & -691309.01 & 2.152915 & $252.0 \pm 2.0$ & González et al. (2005) \\
\hline MACHO 52.5169.24 & 051149.45 & -670545.20 & 167.6350 & $286.24 \pm 0.04$ & Pietrzyński et al. (2013) \\
\hline МАCHO 79.5377.76 & 051323.91 & -692248.90 & 2.636577 & $250.0 \pm 1.0$ & This paper \\
\hline MACHO 2.5509.50 & 051401.91 & -684118.41 & 117.8708 & $265.10 \pm 0.08$ & Pietrzyński et al. (2013) \\
\hline MACHO 79.5500 .60 & 051405.95 & -691556.83 & 771.7806 & $266.38 \pm 0.07$ & Pietrzyński et al. (2013) \\
\hline MACHO 6.5730.3092 & 051541.50 & -700439.00 & 1.761014 & $250.9 \pm 2.6$ & González et al. (2005) \\
\hline MACHO 78.6097.13 & 051804.70 & -694819.02 & 3.107023 & $301.7 \pm 2.4$ & González et al. (2005) \\
\hline HV 12012 & 051911.78 & -694224.38 & 2.727125 & $261.4 \pm 4.6$ & Ribas et al. (2002) \\
\hline MACHO 78.6827.66 & 052235.00 & -693144.01 & 2.183358 & $250.8 \pm 3.0$ & González et al. (2005) \\
\hline MACHO 77.7311.102 & 052525.55 & -693304.49 & 157.3243 & $276.66 \pm 0.06$ & Pietrzyński et al. (2013) \\
\hline MACHO 80.7436.52 & 052604.40 & -691710.99 & 1.664135 & $303.2 \pm 3.8$ & González et al. (2005) \\
\hline TYC 8891-3349-1 & 052606.15 & -671056.98 & 3.30161 & $267 \pm 3$ & Ostrov \& Lapasset (2003) \\
\hline MACHO 80.7438 .42 & 052621.60 & -690545.00 & 1.505947 & $292.7 \pm 4.4$ & González et al. (2005) \\
\hline [L72] LH 54-425 & 052624.25 & -673017.19 & 2.24741 & $300.9 \pm 2.3$ & Williams et al. (2008) \\
\hline HV 2543 & 052727.40 & -671154.55 & 4.829046 & $293.2 \pm 6$ & Ostrov et al. (2000) \\
\hline HV 982 & 052953.00 & -690922.99 & 5.335220 & $287.8 \pm 2.5$ & Fitzpatrick et al. (2002) \\
\hline LMC X-4 & 053249.54 & -662213.30 & 1.40830 & $284.0 \pm 7.0$ & Hutchings et al. (1978) \\
\hline HV 5936 & 053339.03 & -663739.61 & 2.805068 & $314.3 \pm 5.8$ & Fitzpatrick et al. (2003) \\
\hline MACHO 81.8881.21 & 053448.14 & -694236.30 & 4.250806 & $284+3$ & Bonanos $(2009)$ \\
\hline MACHO 81.8763 .8 & 053441.30 & -693139.01 & 1.404740 & $263.1 \pm 3$ & Ostrov (2001) \\
\hline MACHO 81.8881.47 & 053517.57 & -694318.90 & 3.881872 & $272.6 \pm 1.6$ & This paper \\
\hline MACHO 82.9010 .36 & 053550.79 & -691200.44 & 2.762456 & $273.8 \pm 1.2$ & Massey et al. (2012) \\
\hline [HSH95] 38 & 053842.10 & -690607.79 & 3.39 & $275.3 \pm 0.5$ & Massey et al. (2002) \\
\hline [HSH95] 39 & 053842.49 & -690601.29 & 4.06 & $266.9 \pm 0.5$ & Massey et al. (2002) \\
\hline [HSH95] 42 & 053842.18 & -690602.38 & 2.89 & $272.0 \pm 0.5$ & Massey et al. (2002) \\
\hline [HSH95] 77 & 053842.56 & -690604.39 & 1.88 & $275.2 \pm 0.5$ & Massey et al. (2002) \\
\hline LMC X-3 & 053856.63 & -640503.30 & 1.7049 & $310.0 \pm 7.0$ & Cowley et al. (1983) \\
\hline [M2002] LMC 172231 & 053858.10 & -693011.31 & 3.225414 & $271.5 \pm 1.2$ & Massey et al. (2012) \\
\hline LMC X-1 & 053938.84 & -694435.70 & 4.2288 & $221.0 \pm 6.0$ & Hutchings et al. (1987) \\
\hline
\end{tabular}

theoretical model are compared the system appears to be very young $\left(\sim 2 \times 10^{6} \mathrm{yr}\right)$.

In spite of the considerable amount of observational data that has been collected for decades, the absolute dimensions of massive binary components are only known with an accuracy of about 1-3\% (e.g. Clausen 2004). More detailed study based on more precise radial velocities would be very profitable to derive the physical properties of components with higher accuracy. The most promising system for further detailed analysis seems to be HV 2274 because of the putative third component in the system. We still know only a few such systems nowadays, see e.g. Bozkurt \& Değirmenci (2007), while HV 2274 is the first to be discovered out of our Galaxy. Only precise spectral observations and their disentangling should reveal its true nature.

Acknowledgements. We do thank the MACHO and OGLE teams for making all of the observations easily and publicly available. This work was supported by the Research Program MSM0021620860 Physical Study of objects and processes in the Solar System and in Astrophysics of the Ministry of Education of the Czech Republic, by the Czech Science Foundation grant no. P209/10/0715, by the grant UNCE 12 of the Charles University in Prague, and by the grant LG12001 of the Ministry of Education of the Czech Republic. We are also grateful to the ESO team at the La Silla Observatory for their help in maintaining and operating the Danish telescope. The authors would like to thank M. Zejda, J. Liška, J. Janík, and M. Skarka for their important help with photometric observations. G.Michalska is also acknowledged for sending us the unpublished times of minima for selected binaries. The following internet-based resources were used in research for this paper: the SIMBAD database and the VizieR service operated at the CDS, Strasbourg, France, the NASA Astrophysics Data System Bibliographic Services, and the ESO Science Archive Facility.

\section{References}

Bonanos, A. Z. 2009, ApJ, 691, 407

Bozkurt, Z., \& Deǧirmenci, Ö. L. 2007, MNRAS, 379, 370

Claret, A. 1996, A\&A, 315, 415

Claret, A. 2004, A\&A, 424, 919

Claret, A. 2006, A\&A, 453, 769

Clausen, J. V. 2004, New Astron. Rev., 48, 679

Clausen, A., Storm, J., Larsen, S. S., \& Giménez, A. 2003, A\&A, 402, 509

Cowley, A. P., Crampton, D., Hutchings, J. B., Remillard, R., \& Penfold, J. E. 1983, ApJ, 272, 118

Faccioli, L., Alcock, C., Cook, K., et al. 2007, AJ, 134, 1963

Fitzpatrick, E. L., Ribas, I., Guinan, E. F., et al. 2002, ApJ, 564, 260

Fitzpatrick, E. L., Ribas, I., Guinan, E. F., Maloney, F. P., \& Claret, A. 2003, ApJ, 587, 685

Gaposhkin, S. 1970, SAO Special Report, 310

Gaposhkin, S. I. 1977, SAO Special Report, 380

Giménez, A. 1985, ApJ, 297, 405

Giménez, A. 1994, Exp. Astron., 5, 91

Giménez, A., \& García-Pelayo, J. M. 1983, Ap\&SS, 92, 203

González, J. F., Ostrov, P., Morrell, N., \& Minniti, D. 2005, ApJ, 624, 946

Graczyk, D., Soszyński, I., Poleski, R., et al. 2011, AcA, 61, 103

Horn, J., Kubát, J., Harmanec, et al. 1996, A\&A, 309, 521

Hutchings, J. B., Crampton, D., \& Cowley, A. P. 1978, ApJ, 225, 548 
Hutchings, J. B., Crampton, D., Cowley, A. P., Bianchi, L., \& Thompson, I. B. 1987, AJ, 94, 340

Irwin, J. B. 1959, AJ, 64, 149

Kopal, Z. 1978, Dynamics of Close Binary Systems (Dordrecht: Reidel), Astrophys. Space Sci. Lib., 68, 524

Kwee, K. K., \& van Woerden, H. 1956, Bull. Astron. Inst. Netherlands, 12, 327

Leavitt, H. S. 1908, Annals of Harvard College Observatory, 60, 87

Massey, P., Penny, L. R., \& Vukovich, J. 2002, ApJ, 565, 982

Massey, P., Morrell, N. I., Neugent, K. F., et al. 2012, ApJ, 748, 96

Maeder, A. 1999, A\&A, 347, 185

Michalska, G. 2007, IBVS No. 5759

Michalska, G., \& Pigulski, A. 2005, A\&A, 434, 89

Ostrov, P. G. 2001, MNRAS, 321, L25

Ostrov, P. G., \& Lapasset, E. 2003, MNRAS, 338, 141

Ostrov, P. G., Lapasset, E., \& Morrell, N. I. 2000, A\&A, 356, 935

Ostrov, P. G., Morrell, N. I., \& Lapasset, E. 2001, A\&A, 377, 972

Pietrzyński, G., Graczyk, D., Gieren, W., et al. 2013, Nature, 495, 76

Pritchard, J. D., Tobin, W., \& Clark, M. 1994, Exp. Astron., 5, 43
Pritchard, J. D., Tobin, W., Clark, M., \& Guinan, E. F. 1998, MNRAS, 299, 1087 Prša, A., \& Zwitter, T. 2005, ApJ, 628, 426

Reid, W. A., \& Parker, Q. A. 2006, MNRAS, 373, 521

Ribas, I. 2004, New Astron. Rev., 48, 731

Ribas, I., Guinan, E. F., Fitzpatrick, E. L. et al. 2000, ApJ, 528, 692

Ribas, I., Fitzpatrick, E. L., Maloney, F. P., Guinan, E. F., \& Udalski, A. 2002, ApJ, 574, 771

Rohlfs, K., Kreitschmann, J., Feitzinger, J. V., \& Siegman, B. C. 1984, A\&A, 137, 343

Shapley, H., \& Nail, V. McK. 1953, Proc. Nat. Acad. Sci., 39, 1

Škoda, P. 1996, in Astronomical Data Analysis Software and Systems V, eds. G. H. Jacoby, \& J. Barnes, ASP Conf. Ser., 101, 187

van Hamme, W. 1993, AJ, 106, 2096

Watson, R. D., West, S. R. D., Tobin, W., \& Gilmore, A. C. 1992, MNRAS, 528, 527

Williams, S. J., Gies, D. R., Henry, T. J., et al. 2008, ApJ, 682, 492

Wilson, R. E., \& Devinney, E. J. 1971, ApJ, 166, 605

Wozniak, P. R., Vestrand, W. T., Akerlof, C. W., et al. 2004, AJ, 127, 2436

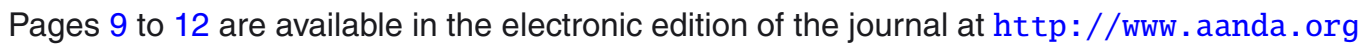




\section{Appendix A: Table of minima}

Table A.1. List of the minima timings used for the analysis.

\begin{tabular}{|c|c|c|c|c|c|}
\hline Star & $\begin{array}{l}\text { JD Hel.- } \\
2400000\end{array}$ & $\begin{array}{l}\text { Error } \\
\text { [day] }\end{array}$ & Epoch & Filter & $\begin{array}{l}\text { Source } \\
\text { Observatory }\end{array}$ \\
\hline HV 982 & 13946.555 & 0.05 & -6634.0 & & Gaposhkin (1977) \\
\hline HV 982 & 17590.584 & 0.05 & -5951.0 & & Gaposhkin (1977) \\
\hline HV 982 & 23875.527 & 0.05 & -4773.0 & & Gaposhkin (1977) \\
\hline HV 982 & 25849.645 & 0.05 & -4403.0 & & Gaposhkin (1977) \\
\hline HV 982 & 26060.243 & 0.10 & -4363.5 & & Gaposhkin (1977) \\
\hline HV 982 & 26412.253 & 0.05 & -4297.5 & & Gaposhkin (1977) \\
\hline HV 982 & 26577.631 & 0.05 & -4266.5 & & Gaposhkin (1977) \\
\hline HV 982 & 27786.315 & 0.05 & -4040.0 & & Gaposhkin (1977) \\
\hline HV 982 & 29189.469 & 0.10 & -3777.0 & & Gaposhkin (1977) \\
\hline HV 982 & 29629.338 & 0.05 & -3694.5 & & Gaposhkin (1977) \\
\hline HV 982 & 31304.630 & 0.05 & -3380.5 & & Gaposhkin (1977) \\
\hline HV 982 & 32070.603 & 0.05 & -3237.0 & & Gaposhkin (1977) \\
\hline HV 982 & 33153.625 & 0.05 & -3034.0 & & Gaposhkin (1977) \\
\hline HV 982 & 49335.3866 & 0.0004 & -1.0 & $u v b y \mathrm{VI}$ & Pritchard et al. (1998) \\
\hline HV 982 & 49337.6668 & 0.0004 & -0.5 & $u v b y \mathrm{VI}$ & Pritchard et al. (1998) \\
\hline HV 982 & 49337.6670 & 0.0010 & -0.5 & uvby & Clausen et al. (2003) \\
\hline HV 982 & 49340.7172 & 0.0005 & 0.0 & uvby & Clausen et al. (2003) \\
\hline HV 982 & 50695.852 & 0.11 & 254.0 & $u v b y \mathrm{VI}$ & Pritchard et al. (1998) \\
\hline HV 982 & 49186.00052 & 0.01299 & -29.0 & $R$ & MACHO, this paper \\
\hline HV 982 & 49188.28011 & 0.00421 & -28.5 & $R$ & MACHO, this paper \\
\hline HV 982 & 49618.14725 & 0.00256 & 52.0 & $R$ & MACHO, this paper \\
\hline HV 982 & 49620.43922 & 0.01275 & 52.5 & $R$ & MACHO, this paper \\
\hline HV 982 & 50023.61929 & 0.00560 & 128.0 & $R$ & MACHO, this paper \\
\hline HV 982 & 50025.92309 & 0.00405 & 128.5 & $R$ & MACHO, this paper \\
\hline HV 982 & 50370.40101 & 0.00352 & 193.0 & $R$ & MACHO, this paper \\
\hline HV 982 & 50367.38712 & 0.00629 & 192.5 & $R$ & MACHO, this paper \\
\hline HV 982 & 50711.84825 & 0.02668 & 257.0 & $R$ & MACHO, this paper \\
\hline HV 982 & 50714.19804 & 0.02646 & 257.5 & $R$ & MACHO, this paper \\
\hline HV 982 & 51277.38263 & 0.01014 & 363.0 & $R$ & MACHO, this paper \\
\hline HV 982 & 51279.71120 & 0.03201 & 363.5 & $R$ & MACHO, this paper \\
\hline HV 982 & 49020.60964 & 0.00588 & -60.0 & $B$ & MACHO, this paper \\
\hline HV 982 & 49017.55154 & 0.00615 & -60.5 & $B$ & MACHO, this paper \\
\hline HV 982 & 49260.69563 & 0.00240 & -15.0 & $B$ & MACHO, this paper \\
\hline HV 982 & 49262.97768 & 0.00197 & -14.5 & $B$ & MACHO, this paper \\
\hline HV 982 & 49490.10034 & 0.00331 & 28.0 & $B$ & MACHO, this paper \\
\hline HV 982 & 49487.05952 & 0.00848 & 27.5 & $B$ & MACHO, this paper \\
\hline HV 982 & 49612.81440 & 0.00315 & 51.0 & $B$ & MACHO, this paper \\
\hline HV 982 & 49609.76750 & 0.00955 & 50.5 & $B$ & MACHO, this paper \\
\hline HV 982 & 49879.57123 & 0.002614 & 101.0 & $B$ & MACHO, this paper \\
\hline HV 982 & 49881.86935 & 0.00480 & 101.5 & $B$ & MACHO, this paper \\
\hline HV 982 & 50231.69273 & 0.00384 & 167.0 & $B$ & MACHO, this paper \\
\hline HV 982 & 50233.99656 & 0.004588 & 167.5 & $B$ & MACHO, this paper \\
\hline HV 982 & 50530.45942 & 0.00416 & 223.0 & $B$ & MACHO, this paper \\
\hline HV 982 & 50532.77709 & 0.00219 & 223.5 & $B$ & MACHO, this paper \\
\hline HV 982 & 51133.32646 & 0.00331 & 336.0 & $B$ & MACHO, this paper \\
\hline HV 982 & 51135.66413 & 0.00742 & 336.5 & $B$ & MACHO, this paper \\
\hline HV 982 & 52293.43371 & 0.01771 & 553.5 & $I$ & OGLE, this paper \\
\hline HV 982 & 52291.04698 & 0.03068 & 553.0 & $I$ & OGLE, this paper \\
\hline HV 982 & 52632.49722 & 0.00320 & 617.0 & $I$ & OGLE, this paper \\
\hline HV 982 & 52634.88640 & 0.00213 & 617.5 & $I$ & OGLE, this paper \\
\hline HV 982 & 53005.95515 & 0.00261 & 687.0 & $I$ & OGLE, this paper \\
\hline HV 982 & 53008.35696 & 0.00726 & 687.5 & $I$ & OGLE, this paper \\
\hline HV 982 & 53355.15161 & 0.00636 & 752.5 & $I$ & OGLE, this paper \\
\hline HV 982 & 53358.07636 & 0.01552 & 753.0 & $I$ & OGLE, this paper \\
\hline HV 982 & 53720.85428 & 0.01008 & 821.0 & $I$ & OGLE, this paper \\
\hline HV 982 & 53717.96464 & 0.02203 & 820.5 & $I$ & OGLE, this paper \\
\hline HV 982 & 54083.65165 & 0.01595 & 889.0 & $I$ & OGLE, this paper \\
\hline HV 982 & 54086.08062 & 0.03 & 889.5 & $I$ & OGLE, this paper \\
\hline HV 982 & 54462.44122 & 0.02 & 960.0 & $I$ & OGLE, this paper \\
\hline HV 982 & 54464.89826 & 0.00320 & 960.5 & $I$ & OGLE, this paper \\
\hline HV 982 & 54793.22040 & 0.00373 & 1022.0 & $I$ & OGLE, this paper \\
\hline HV 982 & 54790.35208 & 0.00774 & 1021.5 & $I$ & OGLE, this paper \\
\hline
\end{tabular}


Table A.1. continued.

\begin{tabular}{|c|c|c|c|c|c|}
\hline Star & $\begin{array}{l}\text { JD Hel.- } \\
2400000\end{array}$ & $\begin{array}{l}\text { Error } \\
\text { [day] }\end{array}$ & Epoch & Filter & $\begin{array}{l}\text { Source } \\
\text { Observatory }\end{array}$ \\
\hline HV 982 & 56257.56416 & 0.0001 & 1296.5 & $I$ & DK154, this paper \\
\hline HV 982 & 56257.56421 & 0.0001 & 1296.5 & $I$ & DK154, this paper \\
\hline HV 982 & 56265.71152 & 0.0001 & 1298.0 & $I$ & DK154, this paper \\
\hline HV 982 & 56265.71137 & 0.0001 & 1298.0 & $I$ & DK154, this paper \\
\hline HV 982 & 56297.72209 & 0.0003 & 1304.0 & $I$ & DK154, this paper \\
\hline HV 982 & 56297.72347 & 0.0003 & 1304.0 & $I$ & DK154, this paper \\
\hline HV 2274 & 13875.807 & & -5977.0 & & Gaposhkin (1977) \\
\hline HV 2274 & 23486.545 & & -4298.5 & & Gaposhkin (1977) \\
\hline HV 2274 & 23638.498 & & -4272.0 & & Gaposhkin (1977) \\
\hline HV 2274 & 23732.679 & & -4255.5 & & Gaposhkin (1977) \\
\hline HV 2274 & 26246.582 & & -3816.5 & & Gaposhkin (1977) \\
\hline HV 2274 & 26710.335 & & -3735.5 & & Gaposhkin (1977) \\
\hline HV 2274 & 26956.619 & & -3692.5 & & Gaposhkin (1977) \\
\hline HV 2274 & 27801.283 & & -3545.0 & & Gaposhkin (1977) \\
\hline HV 2274 & 30589.627 & & -3058.0 & & Gaposhkin (1977) \\
\hline HV 2274 & 31299.629 & & -2934.0 & & Gaposhkin (1977) \\
\hline HV 2274 & 31740.496 & & -2857.0 & & Gaposhkin (1977) \\
\hline HV 2274 & 32058.690 & & -2801.5 & & Gaposhkin (1977) \\
\hline HV 2274 & 32347.570 & & -2751.0 & & Gaposhkin (1977) \\
\hline HV 2274 & 32891.428 & & -2656.0 & & Gaposhkin (1977) \\
\hline HV 2274 & 32940.457 & & -2647.5 & & Gaposhkin (1977) \\
\hline HV 2274 & 48099.818 & & 0.0 & & Watson et al. (1992) \\
\hline HV 2274 & 48102.817 & & 0.5 & & Watson et al. (1992) \\
\hline HV 2274 & 48827.0456 & & 127.0 & & MP05 \\
\hline HV 2274 & 48829.988 & & 127.5 & & MP05 \\
\hline HV 2274 & 48947.28675 & 0.01254 & 148.0 & $B$ & MACHO, this paper \\
\hline HV 2274 & 48950.23969 & 0.01483 & 148.5 & $B$ & $\mathrm{MACHO}$, this paper \\
\hline HV 2274 & 49136.25131 & 0.00567 & 181.0 & $B$ & MACHO, this paper \\
\hline HV 2274 & 49139.18771 & 0.01070 & 181.5 & $B$ & MACHO, this paper \\
\hline HV 2274 & 49359.56433 & 0.00636 & 220.0 & $B$ & $\mathrm{MACHO}$, this paper \\
\hline HV 2274 & 49362.49934 & 0.00401 & 220.5 & $B$ & $\mathrm{MACHO}$, this paper \\
\hline HV 2274 & 49499.9047 & & 244.5 & & MP05 \\
\hline HV 2274 & 49502.7137 & & 245.0 & & MP05 \\
\hline HV 2274 & 49614.42969 & 0.00578 & 264.5 & $B$ & $\mathrm{MACHO}$, this paper \\
\hline HV 2274 & 49617.24018 & 0.00664 & 265.0 & $B$ & $\mathrm{MACHO}$, this paper \\
\hline HV 2274 & 49889.2576 & & 312.5 & & MP05 \\
\hline HV 2274 & 49892.104 & & 313.0 & & MP05 \\
\hline HV 2274 & 49906.44901 & 0.01254 & 315.5 & $B$ & $\mathrm{MACHO}$, this paper \\
\hline HV 2274 & 49909.28085 & 0.01763 & 316.0 & $B$ & MACHO, this paper \\
\hline HV 2274 & 50287.19199 & 0.00452 & 382.0 & $B$ & $\mathrm{MACHO}$, this paper \\
\hline HV 2274 & 50290.07136 & 0.00767 & 382.5 & $B$ & $\mathrm{MACHO}$, this paper \\
\hline HV 2274 & 50659.39741 & 0.00515 & 447.0 & $B$ & MACHO, this paper \\
\hline HV 2274 & 50662.24002 & 0.00425 & 447.5 & $B$ & $\mathrm{MACHO}$, this paper \\
\hline HV 2274 & 50957.1546 & & 499.0 & & MP05 \\
\hline HV 2274 & 50959.971 & & 499.5 & & MP05 \\
\hline HV 2274 & 51214.82801 & 0.00315 & 544.0 & $B$ & MACHO, this paper \\
\hline HV 2274 & 51217.64304 & 0.00527 & 544.5 & $B$ & $\mathrm{MACHO}$, this paper \\
\hline HV 2274 & 52445.93977 & 0.00596 & 759.0 & $I$ & OGLE, this paper \\
\hline HV 2274 & 52448.67936 & 0.01065 & 759.5 & $I$ & OGLE, this paper \\
\hline HV 2274 & 52998.35630 & 0.00504 & 855.5 & $I$ & OGLE, this paper \\
\hline HV 2274 & 53001.37141 & 0.00431 & 856.0 & $I$ & OGLE, this paper \\
\hline HV 2274 & 53528.17437 & 0.00624 & 948.0 & $I$ & OGLE, this paper \\
\hline HV 2274 & 53530.84233 & 0.00424 & 948.5 & $I$ & OGLE, this paper \\
\hline HV 2274 & 54069.07211 & 0.01346 & 1042.5 & $I$ & OGLE, this paper \\
\hline HV 2274 & 54072.15199 & 0.01866 & 1043.0 & $I$ & OGLE, this paper \\
\hline HV 2274 & 54469.86569 & 0.02703 & 1112.5 & $I$ & OGLE, this paper \\
\hline HV 2274 & 54472.97310 & 0.01248 & 1113.0 & $I$ & OGLE, this paper \\
\hline HV 2274 & 54819.14866 & 0.00470 & 1173.5 & $I$ & OGLE, this paper \\
\hline HV 2274 & 54822.26597 & 0.00618 & 1174.0 & $I$ & OGLE, this paper \\
\hline HV 2274 & 56204.78877 & 0.00079 & 1415.5 & $R$ & DK154, this paper \\
\hline HV 2274 & 56267.77936 & 0.00267 & 1426.5 & $R$ & DK154, this paper \\
\hline HV 2274 & 56270.95500 & 0.00257 & 1427.0 & $R$ & DK154, this paper \\
\hline HV 2274 & 56299.58505 & 0.00231 & 1432.0 & $R$ & DK154, this paper \\
\hline HV 2274 & 56290.67754 & 0.00178 & 1430.5 & $R$ & DK154, this paper \\
\hline
\end{tabular}


Table A.1. continued.

\begin{tabular}{|c|c|c|c|c|c|}
\hline Star & $\begin{array}{l}\text { JD Hel.- } \\
2400000\end{array}$ & $\begin{array}{l}\text { Error } \\
\text { [day] }\end{array}$ & Epoch & Filter & $\begin{array}{l}\text { Source } \\
\text { Observatory }\end{array}$ \\
\hline МАCHO 78.6097.13 & 48968.15382 & 0.00342 & -1112.5 & $B R$ & MACHO, this paper \\
\hline МАСНО 78.6097.13 & 48969.63869 & 0.00206 & -1112.0 & $B R$ & MACHO, this paper \\
\hline MACHO 78.6097.13 & 49201.18083 & 0.00205 & -1037.5 & $B R$ & $\mathrm{MACHO}$, this paper \\
\hline MACHO 78.6097.13 & 49202.66301 & 0.00385 & -1037.0 & $B R$ & MACHO, this paper \\
\hline МАCHO 78.6097.13 & 49431.10223 & 0.00306 & -963.5 & $B R$ & MACHO, this paper \\
\hline MACHO 78.6097.13 & 49432.57665 & 0.00549 & -963.0 & $B R$ & $\mathrm{MACHO}$, this paper \\
\hline MACHO 78.6097.13 & 49631.43145 & 0.00707 & -899.0 & $B R$ & MACHO, this paper \\
\hline МАCHO 78.6097.13 & 49633.06235 & 0.00284 & -898.5 & $B R$ & MACHO, this paper \\
\hline МАCHO 78.6097.13 & 49855.13418 & 0.00370 & -827.0 & $B R$ & MACHO, this paper \\
\hline MACHO 78.6097.13 & 49856.77458 & 0.00531 & -826.5 & $B R$ & $\mathrm{MACHO}$, this paper \\
\hline МАCHO 78.6097.13 & 50106.80963 & 0.00559 & -746.0 & $B R$ & MACHO, this paper \\
\hline МАCHO 78.6097.13 & 50108.44539 & 0.00551 & -745.5 & $B R$ & MACHO, this paper \\
\hline МАСНО 78.6097.13 & 50305.65322 & 0.00573 & -682.0 & $B R$ & MACHO, this paper \\
\hline МАCHO 78.6097.13 & 50307.29116 & 0.00378 & -681.5 & $B R$ & MACHO, this paper \\
\hline MACHO 78.6097.13 & 50557.32252 & 0.00262 & -601.0 & $B R$ & MACHO, this paper \\
\hline MACHO 78.6097.13 & 50558.96600 & 0.00634 & -600.5 & $B R$ & MACHO, this paper \\
\hline MACHO 78.6097.13 & 50801.31406 & 0.00683 & -522.5 & $B R$ & MACHO, this paper \\
\hline MACHO 78.6097.13 & 50802.77817 & 0.00996 & -522.0 & $B R$ & MACHO, this paper \\
\hline МАCHO 78.6097.13 & 51231.54291 & 0.00308 & -384.0 & $B R$ & MACHO, this paper \\
\hline MACHO 78.6097.13 & 51233.18484 & 0.00640 & -383.5 & $B R$ & $\mathrm{MACHO}$, this paper \\
\hline MACHO 78.6097.13 & 52424.64757 & 0.00294 & 0.0 & $I$ & OGLE, this paper \\
\hline MACHO 78.6097.13 & 52426.28805 & 0.00233 & 0.5 & $I$ & OGLE, this paper \\
\hline МАCHO 78.6097.13 & 52993.24107 & 0.00798 & 183.0 & I & OGLE, this paper \\
\hline MACHO 78.6097.13 & 52994.86101 & 0.00267 & 183.5 & $I$ & OGLE, this paper \\
\hline MACHO 78.6097.13 & 53524.54977 & 0.00558 & 354.0 & $I$ & OGLE, this paper \\
\hline МАCHO 78.6097.13 & 53523.05361 & 0.00298 & 353.5 & $I$ & OGLE, this paper \\
\hline МАСНО 78.6097.13 & 54270.23860 & 0.00578 & 594.0 & $I$ & OGLE, this paper \\
\hline МАСНО 78.6097.13 & 54271.83416 & 0.00167 & 594.5 & $I$ & OGLE, this paper \\
\hline MACHO 78.6097.13 & 54782.91373 & 0.00432 & 759.0 & $I$ & OGLE, this paper \\
\hline MACHO 78.6097.13 & 54784.48892 & 0.00370 & 759.5 & $I$ & OGLE, this paper \\
\hline MACHO 78.6097.13 & 56314.70125 & 0.00179 & 1252.0 & $R$ & DK154, this paper \\
\hline MACHO 8 & 66 & 5 & -0.5 & & 5 \\
\hline MACHO 81.8881.47 & 49153.34993 & 0.0005 & 0.0 & & MP05 \\
\hline MACHO 81.8881.47 & 49392.29958 & 0.0005 & 61.5 & & MP05 \\
\hline MACHO 81.8881.47 & 49394.04567 & 0.0005 & 62.0 & & MP05 \\
\hline MACHO 81.8881.47 & 49790.01510 & 0.0005 & 164.0 & & MP05 \\
\hline MACHO 81.8881.47 & 49792.12920 & 0.0005 & 164.5 & & MP05 \\
\hline MACHO 81.8881.47 & 50170.45366 & 0.0005 & 262.0 & & MP05 \\
\hline MACHO 81.8881.47 & 50172.52273 & 0.0005 & 262.5 & & MP05 \\
\hline МАCHO 81.8881 .47 & 50341.26363 & 0.0005 & 306.0 & & MP05 \\
\hline МАCHO 81.8881 .47 & 50343.32028 & 0.0005 & 306.5 & & MP05 \\
\hline MACHO 81.8881.47 & 50987.69498 & 0.0005 & 472.5 & & MP05 \\
\hline MACHO 81.8881.47 & 50989.55366 & 0.0005 & 473.0 & & MP05 \\
\hline MACHO 81.8881.47 & 51003.19013 & 0.0005 & 476.5 & & MP05 \\
\hline MACHO 81.8881.47 & 51005.07908 & 0.0005 & 477.0 & & MP05 \\
\hline MACHO 81.8881.47 & 51581.55557 & 0.0005 & 625.5 & & MP05 \\
\hline MACHO 81.8881.47 & 51583.49576 & 0.0005 & 626.0 & & MP05 \\
\hline MACHO 81.8881.47 & 52291.91465 & 0.0091 & 808.5 & $I$ & OGLE, this paper \\
\hline MACHO 81.8881.47 & 52293.91729 & 0.0037 & 809.0 & $I$ & OGLE, this paper \\
\hline MACHO 81.8881.47 & 52635.53595 & 0.0052 & 897.0 & $I$ & OGLE, this paper \\
\hline MACHO 81.8881.47 & 52637.38335 & 0.0877 & 897.5 & $I$ & OGLE, this paper \\
\hline MACHO 81.8881.47 & 53184.70263 & 0.0163 & 1038.5 & $I$ & OGLE, this paper \\
\hline MACHO 81.8881.47 & 53186.77939 & 0.1164 & 1039.0 & $I$ & OGLE, this paper \\
\hline МАCHO 81.8881 .47 & 53912.70392 & 0.0052 & 1226.0 & $I$ & OGLE, this paper \\
\hline МАCHO 81.8881 .47 & 53914.45626 & 0.0034 & 1226.5 & $I$ & OGLE, this paper \\
\hline MACHO 81.8881.47 & 54485.06658 & 0.0014 & 1373.5 & $I$ & OGLE, this paper \\
\hline MACHO 81.8881.47 & 54487.25375 & 0.0049 & 1374.0 & $I$ & OGLE, this paper \\
\hline MACHO 81.8881.47 & 54830.54160 & 0.0042 & 1462.5 & $I$ & OGLE, this paper \\
\hline МАCHO 81.8881.47 & 54832.75781 & 0.0124 & 1463.0 & $I$ & OGLE, this paper \\
\hline MACHO 81.8881.47 & 56284.61939 & 0.0008 & 1837.0 & $R$ & DK154, this paper \\
\hline MACHO 81.8881.47 & 56332.75573 & 0.0010 & 1849.5 & $R$ & DK154, this paper \\
\hline MACHO 79.5377.76 & 6934 & 0.00419 & -12 & $R$ & MACHO, this paper \\
\hline MACHO 79.5377.76 & 48966.92100 & 0.00198 & -1250.0 & $R$ & MACHO, this paper \\
\hline
\end{tabular}


Table A.1. continued.

\begin{tabular}{|c|c|c|c|c|c|}
\hline Star & $\begin{array}{l}\text { JD Hel.- } \\
2400000\end{array}$ & $\begin{array}{l}\text { Error } \\
\text { [day] }\end{array}$ & Epoch & Filter & $\begin{array}{l}\text { Source } \\
\text { Observatory }\end{array}$ \\
\hline MACHO 79.5377.76 & 49197.69453 & 0.00124 & -1162.5 & $R$ & MACHO, this paper \\
\hline МАCHO 79.5377.76 & 49198.94037 & 0.00551 & -1162.0 & $R$ & MACHO, this paper \\
\hline МАCHO 79.5377.76 & 49452.04656 & 0.00129 & -1066.0 & $R$ & MACHO, this paper \\
\hline MACHO 79.5377.76 & 49453.44570 & 0.00382 & -1065.5 & $R$ & MACHO, this paper \\
\hline MACHO 79.5377.76 & 49702.52111 & 0.00264 & -971.0 & $R$ & MACHO, this paper \\
\hline MACHO 79.5377.76 & 49703.92184 & 0.00163 & -970.5 & $R$ & MACHO, this paper \\
\hline МАСНО 79.5377.76 & 49951.76191 & 0.00140 & -876.5 & $R$ & $\mathrm{MACHO}$, this paper \\
\hline MACHO 79.5377.76 & 49950.35515 & 0.00235 & -877.0 & $R$ & MACHO, this paper \\
\hline MACHO 79.5377.76 & 50177.10012 & 0.00171 & -791.0 & $R$ & MACHO, this paper \\
\hline МАCHO 79.5377.76 & 50178.51120 & 0.00206 & -790.5 & $R$ & MACHO, this paper \\
\hline МАCHO 79.5377.76 & 50356.38361 & 0.00169 & -723.0 & $R$ & MACHO, this paper \\
\hline MACHO 79.5377.76 & 50355.15816 & 0.00177 & -723.5 & $R$ & MACHO, this paper \\
\hline MACHO 79.5377.76 & 50626.72815 & 0.00113 & -620.5 & $R$ & MACHO, this paper \\
\hline МАСНО 79.5377.76 & 50627.95207 & 0.00272 & -620.0 & $R$ & MACHO, this paper \\
\hline МАСНО 79.5377.76 & 50952.24739 & 0.00722 & -497.0 & $R$ & MACHO, this paper \\
\hline MACHO 7 & 1.03095 & 0.04825 & -497.5 & $R$ & MACHO, this paper \\
\hline .5377 .76 & 51324.00854 & 0.00419 & -356.0 & $R$ & MACHO, this paper \\
\hline MACHO 79.5377.76 & 51325.41971 & 0.00198 & -355.5 & $R$ & MACHO, this paper \\
\hline MACHO 79.5377.76 & 52447.20093 & 0.00156 & 70.0 & $I$ & OGLE, this paper \\
\hline MACHO 79.5377.76 & 52445.95855 & 0.00132 & 69.5 & $I$ & OGLE, this paper \\
\hline МАСНО 79.5377.76 & 52994.36017 & 0.00348 & 277.5 & $I$ & OGLE, this paper \\
\hline MACHO 79.5377.76 & 52992.97769 & 0.00256 & 277.0 & $I$ & OGLE, this paper \\
\hline MACHO 79.5377.76 & 53354.19440 & 0.00556 & 414.0 & $I$ & OGLE, this paper \\
\hline МАCHO 79.5377.76 & 53352.92752 & 0.01318 & 413.5 & $I$ & OGLE, this paper \\
\hline МАCHO 79.5377.76 & 53892.06597 & 0.00182 & 618.0 & $I$ & OGLE, this paper \\
\hline MACHO 79.5377.76 & 53893.41737 & 0.00150 & 618.5 & $I$ & OGLE, this paper \\
\hline MACHO 79.5377.76 & 54522.21822 & 0.00205 & 857.0 & $I$ & OGLE, this paper \\
\hline МАCHO 79.5377.76 & 54520.91151 & 0.00071 & 856.5 & $I$ & OGLE, this paper \\
\hline МАCHO 79.5377.76 & 54833.34419 & 0.00145 & 975.0 & $I$ & OGLE, this paper \\
\hline МАCHO 79.5377.76 & 54832.01969 & 0.00063 & 974.5 & $I$ & OGLE, this paper \\
\hline МАCHO 79.5377.76 & 56315.12485 & 0.005 & 1537.0 & $R$ & DK154, this paper \\
\hline МАCHO 79.5377.76 & 56383.67777 & 0.00040 & 1563.0 & $R$ & DK154, this paper \\
\hline
\end{tabular}

\section{Appendix B: Table of radial velocities}

Table B.1. List of the radial velocities used for the analysis.

\begin{tabular}{ccrr}
\hline \hline Star & $\begin{array}{c}\text { JD Hel.- } \\
2400000\end{array}$ & $\begin{array}{r}R V_{1} \\
{\left[\mathrm{~km} \mathrm{~s}^{-1}\right]}\end{array}$ & $\begin{array}{r}R V_{2} \\
{\left[\mathrm{~km} \mathrm{~s}^{-1}\right]}\end{array}$ \\
\hline MACHO 81.8881.47 & 52243.62965 & 197.825 & 344.054 \\
MACHO 81.8881.47 & 52243.69480 & 169.847 & 374.850 \\
MACHO 81.8881.47 & 52243.77655 & 157.162 & 397.739 \\
MACHO 81.8881.47 & 52246.54851 & 430.591 & 119.457 \\
MACHO 81.8881.47 & 52257.64245 & 376.268 & 169.630 \\
MACHO 81.8881.47 & 52270.74072 & 208.679 & 322.865 \\
MACHO 81.8881.47 & 52289.69123 & 376.342 & 169.518 \\
MACHO 81.8881.47 & 52294.58185 & 137.413 & 418.659 \\
MACHO 81.8881.47 & 52320.67094 & 391.611 & 143.591 \\
\hline MACHO 79.5377.76 & 52214.79259 & 58.559 & 440.768 \\
MACHO 79.5377.76 & 52226.72978 & 415.303 & 94.430 \\
MACHO 79.5377.76 & 52243.72257 & 38.347 & 451.361 \\
MACHO 79.5377.76 & 52244.58815 & 415.863 & 77.425 \\
MACHO 79.5377.76 & 52244.70911 & 441.078 & 54.155 \\
MACHO 79.5377.76 & 52245.81943 & 121.623 & 372.537 \\
MACHO 79.5377.76 & 52246.60116 & 125.476 & 371.131 \\
MACHO 79.5377.76 & 52270.69835 & 325.508 & 177.316 \\
MACHO 79.5377.76 & 52270.76503 & 352.081 & 147.711 \\
MACHO 79.5377.76 & 52271.61223 & 387.922 & 115.153 \\
MACHO 79.5377.76 & 52271.63592 & 373.100 & 124.104 \\
MACHO 79.5377.76 & 52296.59889 & 91.741 & 409.521 \\
MACHO 79.5377.76 & 52309.54136 & 36.136 & 470.775 \\
MACHO 79.5377.76 & 52309.57452 & 31.116 & 476.893 \\
\hline
\end{tabular}

